\title{
Perspectives and Challenges in Microbial Communities Metabolic Modeling
}

\author{
Emanuele Bosit, Giovanni Baccit, Alessio Mengoni* and Marco Fondi \\ Department of Biology, University of Florence, Florence, Italy
}

Bacteria have evolved to efficiently interact each other, forming complex entities known as microbial communities. These "super-organisms" play a central role in maintaining the health of their eukaryotic hosts and in the cycling of elements like carbon and nitrogen. However, despite their crucial importance, the mechanisms that influence the functioning of microbial communities and their relationship with environmental perturbations are obscure. The study of microbial communities was boosted by tremendous advances in sequencing technologies, and in particular by the possibility to

\section{OPEN ACCESS}

Edited by:

loannis P. Androulakis,

Rutgers University, The State

University of New Jersey,

United States

Reviewed by:

Mitsuyuki Nakao,

Tohoku University, Japan

Osbaldo Resendis-Antonio,

National Autonomous University

of Mexico, Mexico

*Correspondence:

Alessio Mengoni

alessio.mengoni@unifi.it

tThese authors have contributed equally to this work.

Specialty section:

This article was submitted to Systems Biology,

a section of the journal

Frontiers in Genetics

Received: 10 March 2017 Accepted: 09 June 2017

Published: 21 June 2017

Citation:

Bosi E, Bacci G, Mengoni A and

Fondi M (2017) Perspectives and Challenges in Microbial Communities Metabolic Modeling. Front. Genet. 8:88.

doi: 10.3389/fgene.2017.00088 determine genomic sequences of bacteria directly from environmental samples. Indeed, with the advent of metagenomics, it has become possible to investigate, on a previously unparalleled scale, the taxonomical composition and the functional genetic elements present in a specific community. Notwithstanding, the metagenomic approach per se suffers some limitations, among which the impossibility of modeling molecular-level (e.g., metabolic) interactions occurring between community members, as well as their effects on the overall stability of the entire system. The family of constraint-based methods, such as flux balance analysis, has been fruitfully used to translate genome sequences in predictive, genome-scale modeling platforms. Although these techniques have been initially developed for analyzing single, well-known model organisms, their recent improvements allowed engaging in multi-organism in silico analyses characterized by a considerable predictive capability. In the face of these advances, here we focus on providing an overview of the possibilities and challenges related to the modeling of metabolic interactions within a bacterial community, discussing the feasibility and the perspectives of this kind of analysis in the (near) future.

Keywords: microbial communities, metabolic modeling, constraint-based modeling, metabolic interactions, microbiome, mcFBA

\section{METABOLIC-BASED INTERACTIONS AND THE MICROBIOME}

The advent of high-throughput sequencing platforms (NGS) represents one of the most significant milestones in the field of microbial ecology. The possibility of determining genomic sequences directly from environmental samples, circumventing the culturability issues related to most of the bacterial species, allows to investigate the composition of the microbial communities from taxonomical (microbiome) and functional (metagenome) point of view. Metagenomics, in particular, can be used to identify the metabolic potential of a microbial community in terms of the presence of genes encoding enzymes involved in specific metabolic pathways. 
Metabolic interactions are pivotal for maintaining the community processes functions and for structuring the ecology of the host-microbiome unit (Harcombe et al., 2014; Ponomarova and Patil, 2015; Zelezniak et al., 2015). For example, in a community of oceanic plankton the exchange of 2,3dihydroxypropane-1-sulfonate from the diatom Thalassiosira pseudonana to a bacterium from the Roseobacter clade has been demonstrated (Durham et al., 2015). Concerning the hostassociated microbiota, whose implications for human health and development are well established (Lupton, 2004; Sonnenburg et al., 2005; Ley et al., 2006; Frank et al., 2007; Candela et al., 2008; Turnbaugh et al., 2008; Fukuda et al., 2011; Kozyrskyj et al., 2011; Olszak et al., 2012; Yatsunenko et al., 2012), its composition is the result of complex (and poorly understood) interactions which often depends on metabolic effectors occurring at the molecular level between host and microbes, deriving from long-term coadaptation and short-term changes of environmental conditions (as exemplified in the hologenome theory; Theis et al., 2016) In the human gut microbiota, one of the most illuminating examples is fucose, a sugar commonly found as glycan component in epithelial cells of the mammal intestine (Terahara et al., 2011). Glycan fucosylation, induced by the presence of specific symbionts (such as Bacteroides thetaiotaomicron; Bry et al., 1996), has a role in the foraging of commensal bacteria and, consequently, in the stability of gut microbiota. In turn, a fucoseexposed microbiota improves its host health through different mechanisms, such as the production of short chain fatty acids and the inhibition of pathogen colonization (Pham et al., 2014).

\section{TOWARD PREDICTIVE MODELS IN MICROBIAL ECOLOGY}

Microbial communities can be considered "complex adaptive systems” (Song et al., 2014), where individuals and populations interact, giving rise to system's higher-order (emergent) properties. Communities are in fact comprised of a network of spatially distributed agents (cells) that respond concurrently to the actions of others (cells). Thus, the behavior of the system (the community) can arise from a variety of interactions (e.g., mutualism, antagonism, parasitism, etc.) between agents and their local environment. In fact, sociomicrobiology is moving from the analysis of single model systems (e.g., Dictyostelium discoideum, Myxococcus xanthus, Pseudomonas aeruginosa; West et al., 2006) to more complex models, as those related to host-microbe interaction and to microbial consortia (Wyatt et al., 2016). In recent years, the application of conceptual frameworks from market economy theory has become popular, trying to predict the evolution of a microbial community (including the cross-talk of their members) over time (Werner et al., 2014; Tasoff et al., 2015). At the same time, various approaches for mathematical modeling of microbial communities have been applied, including Lotka-Volterra models, evolutionary game models, thermodynamically based models, non-linear regression models, trait-based modeling and stoichiometric modeling (reviewed in Song et al., 2014). Tools for the simulation of microbial community behavior have also been developed (Lardon et al., 2011) which may include interaction between host and the microbiota (as the eGUT http://www.biosciences-labs.bham.ac.uk/kreftlab/eGUT.html) or "simpler" environments resembling a Petri-dish context (Harcombe et al., 2014). In general, modeling of communities may rely on top-down or bottom-up approaches, defined as population-level models (PLMs) and individual-based models (IBMs), whereas PLMs are best applied to homogeneous environments and IBMs are mostly useful when heterogeneous environments are considered (Hellweger et al., 2016). The possible outcomes of a predictive microbial ecology model are many, from biomedicine, environment science, and biotechnology (i.e., metabolic engineering), paving the way for "synthetic ecology" (Zomorrodi and Segre, 2016). In this sense, artificial microbial communities can be designed, being geared toward precise and efficient bio-performances and, at the same time, maintaining the resilience and the complexity of "near" native microbial communities. Under this view, practices such as bacteriotherapy (Cammarota et al., 2014) and extra-terrestrial life support projects (Hendrickx et al., 2006) could be tightly and efficiently programmed.

However, these mechanistic models are mostly based on sharp functional definitions of microbial groups (e.g., glucose utilizers, cellulolytic, methanogens, etc.) that are often difficult to reconcile with detailed microbiological and metagenomic data. Indeed, (i) the very same microbial strain can have many different functional abilities (even contrasting, e.g., nitrogen fixation and denitrification in rhizobia; Delgado et al., 2007), (ii) the same functions may be carried out by phylogenetically distant organisms (e.g., nitrogen fixation), and (iii) the microbial strains of a given species can harbor different metabolic abilities (due to the dispensable genome fraction; Medini et al., 2005). Moreover, other important challenges include the identification of (molecular) interactions of populations as well as the quantification of fluxes of nutrients and energy among individuals and populations (Hanemaaijer et al., 2015). It is then crucial to have accurate descriptions (or predictions) of the metabolic phenotypes expressed by either a given microbial strain or groups of organisms present in the microbiome.

\section{ACCURATE PHENOTYPIC PREDICTIONS WITH CONSTRAINT-BASED METABOLIC MODELING}

The presence of curated repositories integrating biochemical and genetic knowledge (Kegg, Biocyc; Kanehisa and Goto, 2000; Caspi et al., 2016), together with the increased performances of modern genome annotation tools allows reconstructing the metabolic network of an organism from genome sequence data and literature information. More specifically, Genomescale Metabolic network Reconstructions (GEMREs) integrate, by means of a mathematical formal representation, the set of metabolic reactions occurring in the cell, including information concerning metabolites, biochemical constraints and metabolic enzymes encoding genes (Fondi and Liò, 2015a). This is done by drafting (i) the list of the biochemical reactions that the organism 
can carry out (resumed from genome annotation and literature information) together with the constraints of those reaction (e.g., reaction reversibility), (ii) an organism-specific biomass assembly reaction, based on the relative abundancies of biomass constituents, and (iii) inputs and outputs (exchanged fluxes) from and to the external environment. A reconstruction, including all these information, can be exported in a computable format (such as JSON or SBML) and queried with different constraintbased (CB) methods to obtain quantitative predictions of growth phenotypes.

The most commonly used $\mathrm{CB}$ technique is flux balance analysis (FBA) (Orth et al., 2010), which relies on modeling the biochemical system under investigation with a stoichiometric matrix and a flux vector. This is a compact representation of the reactions as a linear system of differential equations, reporting the association between metabolites and reactions together with the corresponding stoichiometric coefficients. Under FBA a pseudosteady state condition is assumed, to let the net sum of production and consumption rates of internal metabolites be 0 . Under this assumption, it is possible to identify a feasible flux of metabolites optimizing a given objective function (e.g., biomass production). The predictions obtained with this approach can, in turn, be used to design targeted experiments and gain insights into the role of genes in different conditions. Moreover, experimental results (such as growth phenotypes, differential expression data, and metabolic profiles) can be easily integrated into the model with well-defined protocols to perform accurate condition- or tissue-specific simulations.

Given the relative simplicity of this kind of analysis and the close relationship with the biology of model organisms, this approach (with slight variations) has been widely used in bioengineering, physiology, and genome-scale synthetic biology (Hjersted et al., 2007; Feist and Palsson, 2008). For example, the yields of economically important cofactors can be predicted in different conditions (Varma and Palsson, 1993), alternative optimal flux distributions can be identified by means of Flux Variability Analysis (Mahadevan and Schilling, 2003), as well as for prediction of pathogenicity (Bosi et al., 2016) and metabolic rewiring in relation to an environmental adaptation (Fondi et al., 2016). Considering the results that can be obtained with such techniques, as the genome sequencing and biochemical characterization of whole microbial communities becomes increasingly more feasible, the application of $\mathrm{CB}$ methods to microbial communities is turning out as a very promising field.

\section{METABOLIC MODELING OF MICROBIAL COMMUNITIES}

In the last years, a number of works describing diverse aspects of multi-organism metabolic modeling has testified the growing interest in this field (Biggs et al., 2015; Heinken and Thiele, 2015). Despite the approximations made when analyzing single organisms become more relevant for community modeling, the possibility of integrating meta-omics data (i.e., metagenomics, transcriptomics, proteomics, metabolomics, and fluxomics) on a highly predictive, systems-based framework allowed gaining important insights into basic aspects of microbial ecology (Fondi and Liò, 2015b). These include the prediction of competition/cooperation patterns (Freilich et al., 2011; Chiu et al., 2014), the characterization of symbiotic interactions (Heinken et al., 2013; Shoaie et al., 2013) and the emergence of community response following nutrient modulations (Zhuang et al., 2011). More practical applications include the prediction of probiotics contrasting Clostridium difficile infections (Steinway et al., 2015), insights into pathogenesis mechanisms (Bordbar et al., 2010) and the metabolic engineering of consortia to achieve optimality in bioremediation or synthetic biology (Brenner et al., 2008; Brune and Bayer, 2012).

A defining feature of community modeling is the sharp increase in complexity with respect to single-organism CB analyses. In other words, the simplistic assumptions at the basis of FBA (i.e., steady-state, biomass production as objective function) become challenging when applied to model multiorganism metabolic interactions. This made necessary the development of innovative approaches, which are briefly described in Table 1. Overall, these methods differ in the scope and complexity of the analyzed community. For instance, dynamics methods based on dFBA are highly predictive for time-resolved analyses, but require a number of parameters which effectively limit their application to small (two or three organisms), well-characterized systems. On the other hand, the enzyme-soup approach relies on simplistic assumptions and limited a priori knowledge of the system under study, making it suited for analyzing complex microbial communities (such as the gut microbiota). Overall, current approaches for community metabolic modeling can be divided into: (i) quantitative methods, having a high predictive potential but being limited to simple systems due to parameterization and/or $a$ priori knowledge required and (ii) large-scale methods, providing mostly qualitative insights but applicable to complex microbial communities.

This simple distinction highlights one current limitation of metabolic modeling methods, that is, the lack of quantitative methods easily scalable to large-scale communities. Although the presence of experimental data (such as meta-omics) can be exploited to improve the biological significance of the predictions obtained in face of the increasing complexity, the development of novel innovative methods overcoming the current limitations is indeed a priority. This includes also (i) the combination of different approaches to obtain hybrid methods optimizing the trade-off between quantitative predictions and scale of the systems and, (ii) the development of integrative frameworks to better combine meta-omics data with metabolic reconstructions. An example of the latter is the dynamic modeling of gut microbiota composition to identify bacteria inhibiting $C$. difficile, performed integrating longitudinal metagenomics data with the network expansion method (Steinway et al., 2015).

Another technical challenge limiting the application of $\mathrm{CB}$ methods to complex communities is the quality of GEMREs that can be used. In fact, prediction of metabolic fluxes maximizing a defined objective function requires high-quality metabolic 
TABLE 1 | Overview of the different approaches adopted to perform metabolic modeling of microbial communities.

\begin{tabular}{|c|c|c|}
\hline Approach & Description & References \\
\hline Compartmentalization & $\begin{array}{l}\text { A logical extension of the multiple compartments for organelles in eukaryotic reconstructions. This approach } \\
\text { combines multiple GEMREs in a single large stoichiometric matrix, defining a compartment for each } \\
\text { organism and transport reactions for the shared metabolites. The objective function used in this case is a } \\
\text { linear combination of the individual biomass functions. }\end{array}$ & $\begin{array}{l}\text { Stolyar et al., 2007; } \\
\text { Bordbar et al., 2010, } \\
\text { Klitgord and Segre, 2010; } \\
\text { Shoaie et al., } 2013\end{array}$ \\
\hline $\begin{array}{l}\text { Community } \\
\text { objectives }\end{array}$ & $\begin{array}{l}\text { This strategy, which is implemented in the OptCom tool, extends the Compartmentalization approach } \\
\text { introducing an objective function designed at the community level. This allows to effectively model trophic } \\
\text { interactions (e.g., commensalism, parasitism, mutualism, etc.) between members of the community, via a } \\
\text { series of nested, bi-level optimizations. }\end{array}$ & $\begin{array}{l}\text { Zomorrodi and Maranas, } \\
\text { 2012; Shoaie et al., 2013, } \\
\text { El-Semman et al., } 2014\end{array}$ \\
\hline $\begin{array}{l}\text { Dynamic } \\
\text { analysis }\end{array}$ & $\begin{array}{l}\text { Instead of using FBA (whose central assumption is the steady state condition), this dynamic approach relies } \\
\text { on dFBA, which allows compounds being accumulated or depleted. Instead of producing static "snapshot" } \\
\text { of the metabolic states, the dFBA framework provides a dynamic description of the adaptation to changing } \\
\text { conditions and nutrients availability. To cope with this totally different framework, a modified version of } \\
\text { OptCom has been tailored to carry out dynamic analyses (dOptCom). Despite the interesting results } \\
\text { obtained with this approach, the application of dFBA is severely hindered by two factors: (i) it is } \\
\text { computationally demanding and (ii) it requires some kinetic parameters (e.g., for growth-limiting } \\
\text { metabolites). A major consequence is the reduced scale of the system that can be analyzed with this } \\
\text { approach, with respect to other methods. }\end{array}$ & $\begin{array}{l}\text { Tzamali et al., 2011; } \\
\text { Zhuang et al., 2011, Hanly } \\
\text { et al., 2012; Chiu et al., } \\
\text { 2014, Hanly and Henson, } \\
\text { 2014; Harcombe et al., } \\
2014\end{array}$ \\
\hline $\begin{array}{l}\text { Spatially } \\
\text { resolved }\end{array}$ & $\begin{array}{l}\text { This approach introduces the study of bacterial spatial diffusion and the resulting structure of (simple) } \\
\text { microbial communities. COMETS, for example, uses dynamic flux balance analysis (dFBA) to perform } \\
\text { time-dependent metabolic simulations of microbial ecosystems, bridging the gap between stoichiometric } \\
\text { and environmental modeling. }\end{array}$ & $\begin{array}{l}\text { Gorochowski et al., 2012; } \\
\text { Harcombe et al., } 2014 \text {, } \\
\text { Phalak et al., } 2016\end{array}$ \\
\hline Enzyme soup & $\begin{array}{l}\text { Radically different from the other methods, the enzyme-soup approach completely neglects any } \\
\text { inter-organism boundary concept. Reactions are not assigned to different species, as the whole community } \\
\text { is treated as a "soup" of enzymes. Since a number of biomass components are shared in the community, } \\
\text { the biomass function has a generalized formulation, representing the biomass of the whole community. In } \\
\text { accordance with its premises, this approach focuses on depicting the metabolic potential of microbial } \\
\text { communities, bypassing the problem of inter-organism interactions. Due to the simple nature of its } \\
\text { assumptions, this method can be easily applied to large complex communities, given the experimental } \\
\text { support of meta-omic data. }\end{array}$ & $\begin{array}{l}\text { Taffs et al., 2009; } \\
\text { Tobalina et al., } 2015\end{array}$ \\
\hline Graph-based & $\begin{array}{l}\text { Methods defined as graph-based have been used to identify competition or cooperation patterns between } \\
\text { bacteria. According to this framework, the stoichiometric matrix is used to generate graph connecting } \\
\text { metabolites, with edges directed from substrates to products. Nodes with in-degree/out-degree ratio equal } \\
\text { to } 0 \text { represent metabolites (seeds) which are consumed but not produced, and therefore must be supplied } \\
\text { to the network. The assessment of seed sets for multiple organisms allows to evaluate the metabolic basis } \\
\text { of competition/cooperation. Since inferences are made regardless of stoichiometry and flux analysis, this } \\
\text { approach shows a remarkable robustness when applied to poor-quality reconstructions, which might affect } \\
\text { conclusions made using FBA-based methods. }\end{array}$ & $\begin{array}{l}\text { Borenstein et al., 2008; } \\
\text { Levy and Borenstein, } 2013\end{array}$ \\
\hline $\begin{array}{l}\text { Network } \\
\text { expansion }\end{array}$ & $\begin{array}{l}\text { This method encompasses an agglomerative algorithm (Network Expansion), which iteratively add reactions } \\
\text { to an initial set of reactions/metabolites, aiming at identifying emergent properties of the growing metabolic } \\
\text { network. The algorithm has been adapted to suit the case of microbial community analysis, studying the } \\
\text { properties of pairwise combinations of bacteria. Basically, starting from an initial set of reactions from both } \\
\text { the microbes, this method iteratively expands the network with a pool of reactions from both organisms, } \\
\text { under the assumptions that metabolic intermediates can be shared. The application of this method allowed } \\
\text { to identify emergent biosynthetic capacities for a large number of bacterial pairs. }\end{array}$ & $\begin{array}{l}\text { Handorf et al., 2005; } \\
\text { Christian et al., 2007, } \\
\text { Steinway et al., } 2015\end{array}$ \\
\hline
\end{tabular}

reconstructions (generated with precise protocols; Thiele and Palsson, 2010) to achieve consistency with the actual biology of the organisms accounted by the reconstruction. However, the model typically requires further refinement (such as integration of literature and/or extant physiological data to identify potential gaps) and validation steps, which can be quite time (and resource) consuming. Resultantly, the protocol used to obtain GEMREs of single organisms cannot be extended to large datasets due to the long time required to carry out these analyses and/or potential knowledge gaps for some organisms (such as unculturable bacteria) hindering the application of bottom-up reconstruction approaches.

General strategies have been developed to rapidly obtain GEMREs for many organisms. These are based on automatic reconstruction from genomes (or binned metagenomic contigs), or comparative approaches relying on orthologous genes with "reference" organisms for which high-quality GEMREs are available. Either way, the obtained draft-quality GEMREs require additional refinement steps to fill potential reaction gaps. Perhaps the most notable example of such largescale analysis is the metabolic reconstruction of 773 human gut microbes using a semi-automatic comparative metabolic reconstruction method (Magnusdottir et al., 2017). Although the analysis of these GEMREs revealed good consistency with known functional features of gut microbiota (e.g., carbon source compounds degradation; Flint et al., 2012), the authors specified the infeasibility of this approach to recover (accurate) quantitative predictions, due to the absence of 
species/condition-specific information (i.e., the breakdown of biomass components). On the other hand, qualitative insights such as prediction of growth-supporting media seem to be less affected by this kind of approximations (Feist and Palsson, 2010).

Altogether, this points to the need of (i) established protocols (such as Thiele and Palsson, 2010) to develop and curate GEMREs for large-scale datasets and (ii) public resources to facilitate this task (see Magnusdottir et al., 2017). In particular, we specifically stress the lack of a data repository describing the biomass composition of different organisms in a variety of conditions. Indeed, such knowledge could be easily integrated in existing reconstruction pipelines and would allow obtaining more biologically relevant GEMREs.

\section{STRENGTHS AND WEAKNESSES OF COMMUNITY MODELS}

Knowledge-driven metabolic engineering of bacterial communities is an emerging field which might shed light on some of the most puzzling biological questions regarding clinical problems (e.g., drug-bacteria interactions; Ye et al., 2014), industrial production design (e.g., enhancing secondary metabolites production; Kim et al., 2016), and environmental safety/health (e.g., bioremediation; Rein et al., 2016) (Figure 1). Several efforts have been directed at characterizing the interactions between bacterial pathogens and their host, aiming at designing probiotic formulations to recover damaged communities (such as the human gut microbiota following C. difficile infection; Buffie et al., 2015), or able to directly suppress pathogen proliferation (Buffie et al., 2015). The metabolic repertoire shared by complex bacterial communities, such as those living in the human gut, has been explored using semi-automated approaches to reconstruct a large set of metabolic models intertwining genomic, metagenomic, and metabolic information (Magnusdottir et al., 2017). Microbial consortia can, in principle, perform complex reactions requiring multiple steps that can be cell- or community-specific (Brenner et al., 2008). Understanding the communication systems underpinning bacterial communities represents a crucial step for the rational design of microbial consortia able to maximize the production of different compounds or for the production of hybrid communities, composed of natural and engineered bacteria, to be used in bioremediation processes (Brune and Bayer, 2012). Despite all the advances made in the integration of omics data into community-level models, more work is needed to overcome limitations imposed by current computational and experimental procedures.

Rational design of engineered microbial communities can be translated into specific services (e.g., bioremediation, metabolites productions, protection against pathogens, etc.). However, this requires the precise annotation of metabolic functions to the species present in the communities, and this can be not feasible, especially when complete and annotated genomes are missing. Despite the recent progress in genome reconstruction from metagenomes (Nielsen et al., 2014), the gap between omics information acquired and reference genomes assembled and available in public databases is still to be filled. Methods for binning metagenomic sequences into taxonomic groups are mainly based on the different DNA composition (i.e., unsupervised methods combining $k$-mer frequencies with coverage information; Alneberg et al., 2014) or on pairwise comparisons with taxonomic annotated sequences (i.e., supervised methods based on sequence similarity; Brady and Salzberg, 2009; Wood and Salzberg, 2014). Even if these methods can be used for partitioning genomes into different "biological units," thus allowing metabolic models reconstruction, at present it is not feasible to recover all the genomes that compose an entire natural community and, consequently, perform metabolic modeling of the whole microbial community. Another bottleneck in community level metabolic modeling is the generation of a model for each component of the community. Indeed, microbial consortia are composed by thousands of strains and producing a different, curated and reliable model for each strain would be very demanding in terms of costs and time (Figure 1). For this reason, the automatic generation of models from genomic and metagenomics data is a mandatory step to increase the resolution power of the community model, especially in natural environment where, in principle, every single cell takes part in maintaining the homeostasis of a particular niche.

On the other hand, while genomics and metagenomics have provided many insights into the role of bacteria in determining potential functional features of a given environment, they both provide a static snapshot of a community, thus failing to deliver a dynamic and fully functional representation. Consequently, obtaining an accurate dynamic model of a community would require longitudinal metagenomics or, more in general, methods to infer growth dynamics of single bacterial species from metagenomics (Korem et al., 2015). However, this might not be straight-forward, since timeresolved metagenomics shows that some species can grow faster than others increasing their abundance quickly enough to significantly change the whole community structure (Bacci et al., 2015). Therefore, given the paradigm of CB metabolic modeling, this could effectively represent a problem when trying to infer the metabolic phenotype of a microbial community. Similarly, it is unlikely that all the members of a microbial consortium are optimally geared toward biomass production. As a consequence, the steady-state assumption (that is the foundation of FBA analyses) may not hold true during simulations.

All these factors force to discuss about which assumptions made in the context of single-organism metabolic modeling can be still tolerated when trying to accurately infer the (metabolic) dynamic of a bacterial community. Indeed, each framework proposed hitherto takes into account different aspects of microbial interactions leaving to researchers the burden of choosing the kind of model that best fits their needs; this decision should be primarily based on the information available as well as on the resolution level that is possible to achieve (Figure 1). As 'omic sciences become more and more affordable and sensible, 


\section{Case selection}
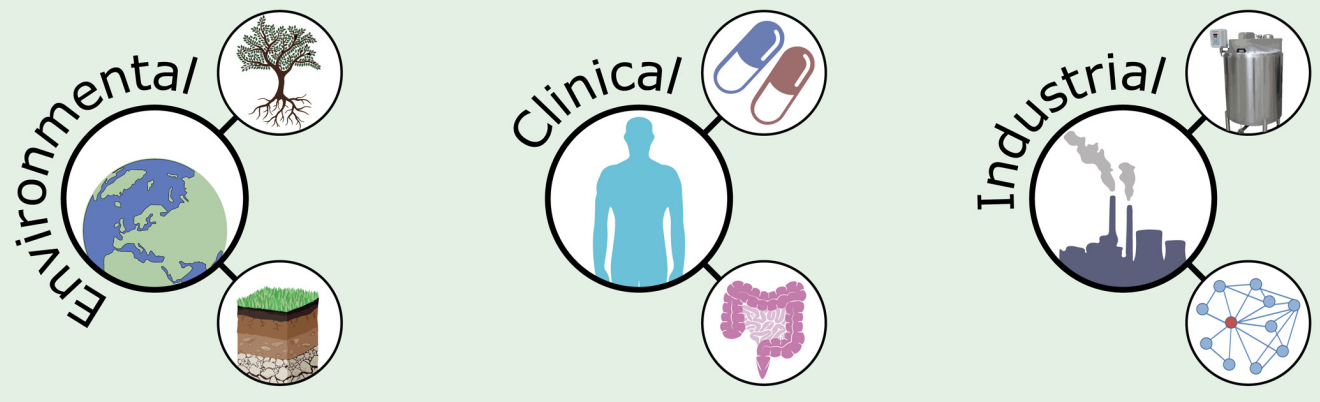

\section{Framework definition}

- Number of organisms thought to interact

- Type of interactions involved

- Steady state condition

- Data availability

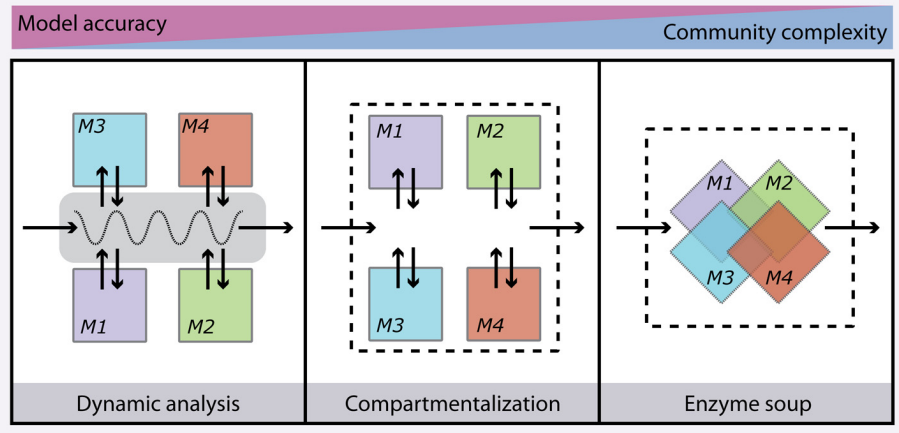

\section{Modelling}

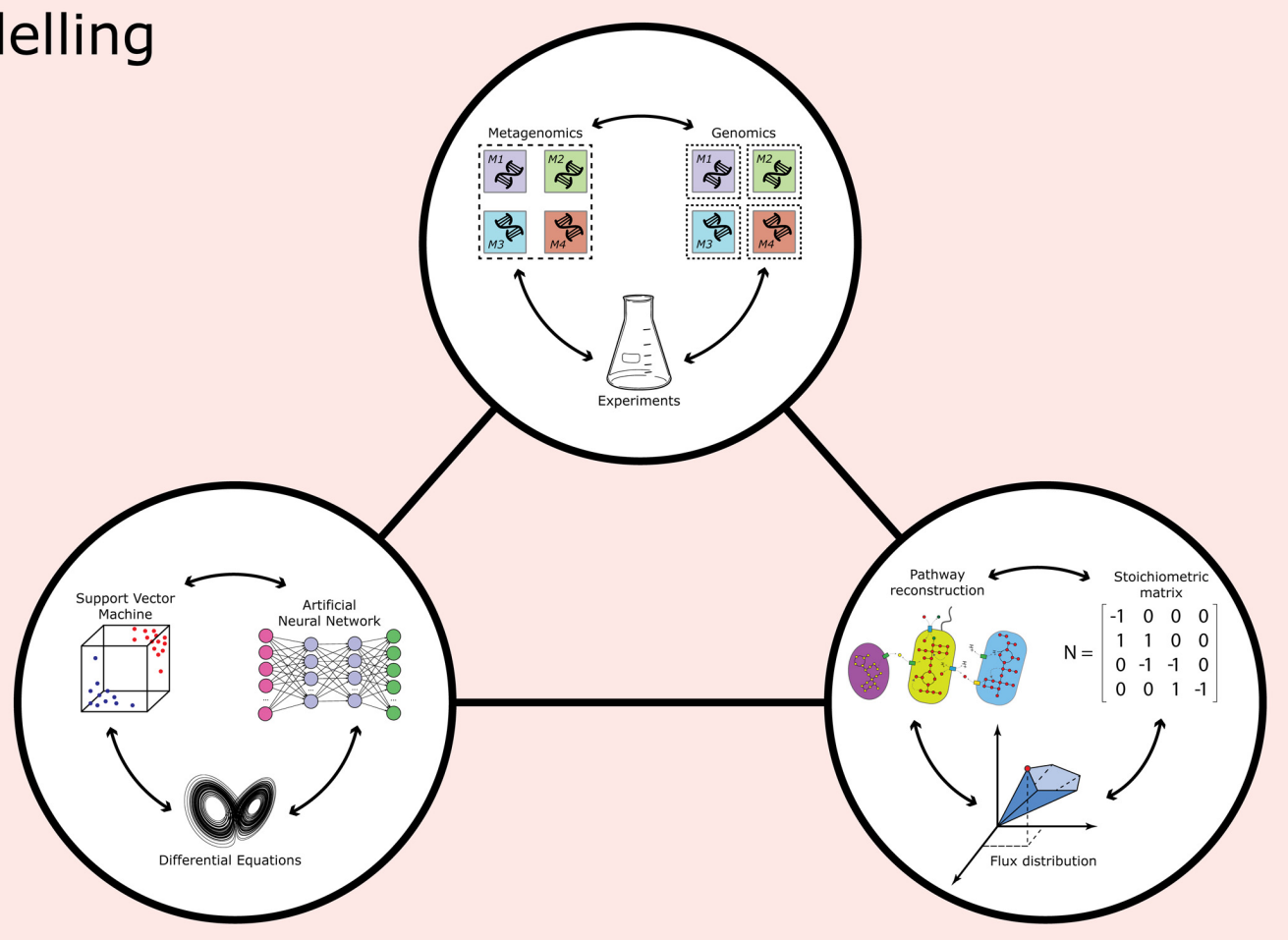

FIGURE 1 | Overview of the main steps and applications in bacterial community metabolic modeling. Microbiomes with environmental, clinical, and industrial relevance (top panel) are selected and models are chosen based on a balance between the desidered model accuracy and the complexity of the microbiome (central panel). Then modeling is applied and combined with information from metagenomic data and genome sequences (and phenotypic/biochemical information) from cultivated microorganisms (lower panel). 
their integration into community-level metabolic models is mandatory to achieve a systems level understanding of these biological entities. This highlights the necessity of a working scheme designed to handle large-scale, community-level reconstructions and to derive quantitative insights.

\section{REFERENCES}

Alneberg, J., Bjarnason, B. S., De Bruijn, I., Schirmer, M., Quick, J., Ijaz, U. Z., et al. (2014). Binning metagenomic contigs by coverage and composition. Nat. Methods 11, 1144-1146. doi: 10.1038/nmeth.3103

Bacci, G., Ceccherini, M., Bani, A., Bazzicalupo, M., Castaldini, M., Galardini, M., et al. (2015). Exploring the dynamics of bacterial community composition in soil: the pan-bacteriome approach. Antonie Van Leeuwenhoek 107, 785-797. doi: 10.1007/s10482-014-0372-4

Biggs, M. B., Medlock, G. L., Kolling, G. L., and Papin, J. A. (2015). Metabolic network modeling of microbial communities. Wiley Interdiscip. Rev. Syst. Biol. Med. 7, 317-334. doi: 10.1002/wsbm.1308

Bordbar, A., Lewis, N. E., Schellenberger, J., Palsson, B. O., and Jamshidi, N. (2010). Insight into human alveolar macrophage and $M$. tuberculosis interactions via metabolic reconstructions. Mol. Syst. Biol. 6:422. doi: 10.1038/msb.2010.68

Borenstein, E., Kupiec, M., Feldman, M. W., and Ruppin, E. (2008). Large-scale reconstruction and phylogenetic analysis of metabolic environments. Proc. Natl. Acad. Sci. U.S.A. 105, 14482-14487. doi: 10.1073/pnas.0806162105

Bosi, E., Monk, J. M., Aziz, R. K., Fondi, M., Nizet, V., and Palsson, B. O. (2016). Comparative genome-scale modelling of Staphylococcus aureus strains identifies strain-specific metabolic capabilities linked to pathogenicity. Proc. Natl. Acad. Sci. U.S.A. 113, E3801-E3809. doi: 10.1073/pnas.1523199113

Brady, A., and Salzberg, S. L. (2009). Phymm and PhymmBL: metagenomic phylogenetic classification with interpolated Markov models. Nat. Methods 6, 673-676. doi: 10.1038/nmeth.1358

Brenner, K., You, L., and Arnold, F. H. (2008). Engineering microbial consortia: a new frontier in synthetic biology. Trends Biotechnol. 26, 483-489. doi: 10.1016/ j.tibtech.2008.05.004

Brune, K. D., and Bayer, T. (2012). Engineering microbial consortia to enhance biomining and bioremediation. Front. Microbiol. 3:203. doi: 10.3389/fmicb. 2012.00203

Bry, L., Falk, P. G., Midtvedt, T., and Gordon, J. I. (1996). A model of host-microbial interactions in an open mammalian ecosystem. Science 273, 1380-1383. doi: 10.1126/science.273.5280.1380

Buffie, C. G., Bucci, V., Stein, R. R., Mckenney, P. T., Ling, L., Gobourne, A., et al. (2015). Precision microbiome reconstitution restores bile acid mediated resistance to Clostridium difficile. Nature 517, 205-208. doi: 10.1038/ nature 13828

Cammarota, G., Ianiro, G., and Gasbarrini, A. (2014). Fecal microbiota transplantation for the treatment of Clostridium difficile infection: a systematic review. J. Clin. Gastroenterol. 48, 693-702. doi: 10.1097/MCG. 0000000000000046

Candela, M., Perna, F., Carnevali, P., Vitali, B., Ciati, R., Gionchetti, P., et al. (2008). Interaction of probiotic Lactobacillus and Bifidobacterium strains with human intestinal epithelial cells: adhesion properties, competition against enteropathogens and modulation of IL-8 production. Int. J. Food Microbiol. 125, 286-292. doi: 10.1016/j.ijfoodmicro.2008.04.012

Caspi, R., Billington, R., Ferrer, L., Foerster, H., Fulcher, C. A., Keseler, I. M., et al. (2016). The MetaCyc database of metabolic pathways and enzymes and the BioCyc collection of pathway/genome databases. Nucleic Acids Res. 44, D471-D480. doi: 10.1093/nar/gkv1164

Chiu, H. C., Levy, R., and Borenstein, E. (2014). Emergent biosynthetic capacity in simple microbial communities. PLoS Comput. Biol. 10:e1003695. doi: 10.1371/ journal.pcbi.1003695

Christian, N., Handorf, T., and Ebenhoh, O. (2007). Metabolic synergy: increasing biosynthetic capabilities by network cooperation. Genome Inform. 18, 320-329. doi: 10.1142/9781860949920_0031

Delgado, M. J., Casella, S., and Bedmar, E. J. (2007). “Denitrification in rhizobialegume symbiosis," in Biology of the Nitrogen Cycle, eds H. Bothe, S. J. Ferguson, and W. E. Newton (Amsterdam: Elsevier Science), 57-66.

\section{AUTHOR CONTRIBUTIONS}

All the authors contributed in conceiving, preparing and revising the manuscript. All the authors approved the manuscript and agreed to be accountable for all aspects of the presented work.

Durham, B. P., Sharma, S., Luo, H., Smith, C. B., Amin, S. A., Bender, S. J., et al. (2015). Cryptic carbon and sulfur cycling between surface ocean plankton. Proc. Natl. Acad. Sci. U.S.A. 112, 453-457. doi: 10.1073/pnas.1413137112

El-Semman, I. E., Karlsson, F. H., Shoaie, S., Nookaew, I., Soliman, T. H., and Nielsen, J. (2014). Genome-scale metabolic reconstructions of Bifidobacterium adolescentis L2-32 and Faecalibacterium prausnitzii A2-165 and their interaction. BMC Syst. Biol. 8:41. doi: 10.1186/1752-0509-8-41

Feist, A. M., and Palsson, B. O. (2008). The growing scope of applications of genome-scale metabolic reconstructions using Escherichia coli. Nat. Biotechnol. 26, 659-667. doi: 10.1038/nbt1401

Feist, A. M., and Palsson, B. O. (2010). The biomass objective function. Curr. Opin. Microbiol. 13, 344-349. doi: 10.1016/j.mib.2010.03.003

Flint, H. J., Scott, K. P., Duncan, S. H., Louis, P., and Forano, E. (2012). Microbial degradation of complex carbohydrates in the gut. Gut Microbes 3, 289-306. doi: $10.4161 /$ gmic. 19897

Fondi, M., Bosi, E., Presta, L., Natoli, D., and Fani, R. (2016). Modelling microbial metabolic rewiring during growth in a complex medium. BMC Genomics 17:970. doi: 10.1186/s12864-016-3311-0

Fondi, M., and Liò, P. (2015a). "Genome-scale metabolic network reconstruction," in Bacterial Pangenomics: Methods and Protocols, eds A. Mengoni, M. Galardini, and M. Fondi (New York, NY: Springer), 233-256.

Fondi, M., and Liò, P. (2015b). Multi -omics and metabolic modelling pipelines: challenges and tools for systems microbiology. Microbiol. Res. 171, 52-64. doi: 10.1016/j.micres.2015.01.003

Frank, D. N., St Amand, A. L., Feldman, R. A., Boedeker, E. C., Harpaz, N., and Pace, N. R. (2007). Molecular-phylogenetic characterization of microbial community imbalances in human inflammatory bowel diseases. Proc. Natl. Acad. Sci. U.S.A. 104, 13780-13785. doi: 10.1073/pnas.0706625104

Freilich, S., Zarecki, R., Eilam, O., Segal, E. S., Henry, C. S., Kupiec, M., et al. (2011). Competitive and cooperative metabolic interactions in bacterial communities. Nat. Commun. 2:589. doi: 10.1038/ncomms1597

Fukuda, S., Toh, H., Hase, K., Oshima, K., Nakanishi, Y., Yoshimura, K., et al. (2011). Bifidobacteria can protect from enteropathogenic infection through production of acetate. Nature 469, 543-547. doi: 10.1038/nature09646

Gorochowski, T. E., Matyjaszkiewicz, A., Todd, T., Oak, N., Kowalska, K., Reid, S., et al. (2012). BSim: an agent-based tool for modeling bacterial populations in systems and synthetic biology. PLOS ONE 7:e42790. doi: 10.1371/journal.pone. 0042790

Handorf, T., Ebenhoh, O., and Heinrich, R. (2005). Expanding metabolic networks: scopes of compounds, robustness, and evolution. J. Mol. Evol. 61, 498-512. doi: 10.1007/s00239-005-0027-1

Hanemaaijer, M., Roling, W. F., Olivier, B. G., Khandelwal, R. A., Teusink, B., and Bruggeman, F. J. (2015). Systems modeling approaches for microbial community studies: from metagenomics to inference of the community structure. Front. Microbiol. 6:213. doi: 10.3389/fmicb.2015.00213

Hanly, T. J., and Henson, M. A. (2014). Dynamic model-based analysis of furfural and HMF detoxification by pure and mixed batch cultures of S. cerevisiae and S. stipitis. Biotechnol. Bioeng. 111, 272-284. doi: 10.1002/bit. 25101

Hanly, T. J., Urello, M., and Henson, M. A. (2012). Dynamic flux balance modeling of $S$. cerevisiae and E. coli co-cultures for efficient consumption of glucose/xylose mixtures. Appl. Microbiol. Biotechnol. 93, 2529-2541. doi: 10.1007/s00253-011-3628-1

Harcombe, W. R., Riehl, W. J., Dukovski, I., Granger, B. R., Betts, A., Lang, A. H., et al. (2014). Metabolic resource allocation in individual microbes determines ecosystem interactions and spatial dynamics. Cell Rep. 7, 1104-1115. doi: 10.1016/j.celrep.2014.03.070

Heinken, A., Sahoo, S., Fleming, R. M., and Thiele, I. (2013). Systems-level characterization of a host-microbe metabolic symbiosis in the mammalian gut. Gut Microbes 4, 28-40. doi: 10.4161/gmic.22370 
Heinken, A., and Thiele, I. (2015). Systems biology of host-microbe metabolomics. Wiley Interdiscip. Rev. Syst. Biol. Med. 7, 195-219. doi: 10.1002/wsbm.1301

Hellweger, F. L., Clegg, R. J., Clark, J. R., Plugge, C. M., and Kreft, J. U. (2016). Advancing microbial sciences by individual-based modelling. Nat. Rev. Microbiol. 14, 461-471. doi: 10.1038/nrmicro.2016.62

Hendrickx, L., De Wever, H., Hermans, V., Mastroleo, F., Morin, N., Wilmotte, A., et al. (2006). Microbial ecology of the closed artificial ecosystem MELiSSA (Micro-Ecological Life Support System Alternative): reinventing and compartmentalizing the Earth's food and oxygen regeneration system for longhaul space exploration missions. Res. Microbiol. 157, 77-86. doi: 10.1016/j. resmic.2005.06.014

Hjersted, J. L., Henson, M. A., and Mahadevan, R. (2007). Genome-scale analysis of Saccharomyces cerevisiae metabolism and ethanol production in fed-batch culture. Biotechnol. Bioeng. 97, 1190-1204. doi: 10.1002/bit. 21332

Kanehisa, M., and Goto, S. (2000). KEGG: kyoto encyclopedia of genes and genomes. Nucleic Acids Res. 28, 27-30. doi: 10.1093/nar/28.1.27

Kim, H. U., Charusanti, P., Lee, S. Y., and Weber, T. (2016). Metabolic engineering with systems biology tools to optimize production of prokaryotic secondary metabolites. Nat. Prod. Rep. 33, 933-941. doi: 10.1039/c6np00019c

Klitgord, N., and Segre, D. (2010). Environments that induce synthetic microbial ecosystems. PLoS Comput. Biol. 6:e1001002. doi: 10.1371/journal.pcbi.1001002

Korem, T., Zeevi, D., Suez, J., Weinberger, A., Avnit-Sagi, T., PompanLotan, M., et al. (2015). Growth dynamics of gut microbiota in health and disease inferred from single metagenomic samples. Science 349, 1101-1106. doi: $10.1126 /$ science.aac 4812

Kozyrskyj, A. L., Bahreinian, S., and Azad, M. B. (2011). Early life exposures: impact on asthma and allergic disease. Curr. Opin. Allergy Clin. Immunol. 11, 400-406. doi: 10.1097/ACI.0b013e328349b166

Lardon, L. A., Merkey, B. V., Martins, S., Dotsch, A., Picioreanu, C., Kreft, J. U., et al. (2011). iDynoMiCS: next-generation individual-based modelling of biofilms. Environ. Microbiol. 13, 2416-2434. doi: 10.1111/j.1462-2920.2011. 02414.x

Levy, R., and Borenstein, E. (2013). Metabolic modeling of species interaction in the human microbiome elucidates community-level assembly rules. Proc. Natl. Acad. Sci. U.S.A. 110, 12804-12809. doi: 10.1073/pnas.1300926110

Ley, R. E., Turnbaugh, P. J., Klein, S., and Gordon, J. I. (2006). Microbial ecology: human gut microbes associated with obesity. Nature 444, 1022-1023. doi: 10. $1038 / 4441022 \mathrm{a}$

Lupton, J. R. (2004). Microbial degradation products influence colon cancer risk: the butyrate controversy. J. Nutr. 134, 479-482.

Magnusdottir, S., Heinken, A., Kutt, L., Ravcheev, D. A., Bauer, E., Noronha, A., et al. (2017). Generation of genome-scale metabolic reconstructions for 773 members of the human gut microbiota. Nat. Biotechnol. 35, 81-89. doi: 10.1038/ nbt. 3703

Mahadevan, R., and Schilling, C. H. (2003). The effects of alternate optimal solutions in constraint-based genome-scale metabolic models. Metab. Eng. 5, 264-276. doi: 10.1016/j.ymben.2003.09.002

Medini, D., Donati, C., Tettelin, H., Masignani, V., and Rappuoli, R. (2005). The microbial pan-genome. Curr. Opin. Genet. Dev. 15, 589-594. doi: 10.1016/j.gde. 2005.09.006

Nielsen, H. B., Almeida, M., Juncker, A. S., Rasmussen, S., Li, J., Sunagawa, S., et al. (2014). Identification and assembly of genomes and genetic elements in complex metagenomic samples without using reference genomes. Nat. Biotechnol. 32, 822-828. doi: 10.1038/nbt.2939

Olszak, T., An, D., Zeissig, S., Vera, M. P., Richter, J., Franke, A., et al. (2012). Microbial exposure during early life has persistent effects on natural killer $\mathrm{T}$ cell function. Science 336, 489-493. doi: 10.1126/science.1219328

Orth, J. D., Thiele, I., and Palsson, B. O. (2010). What is flux balance analysis? Nat. Biotechnol. 28, 245-248. doi: 10.1038/nbt.1614

Phalak, P., Chen, J., Carlson, R. P., and Henson, M. A. (2016). Metabolic modeling of a chronic wound biofilm consortium predicts spatial partitioning of bacterial species. BMC Syst. Biol. 10:90. doi: 10.1186/s12918-016-0334-8

Pham, T. A., Clare, S., Goulding, D., Arasteh, J. M., Stares, M. D., Browne, H. P., et al. (2014). Epithelial IL-22RA1-mediated fucosylation promotes intestinal colonization resistance to an opportunistic pathogen. Cell Host Microbe 16, 504-516. doi: 10.1016/j.chom.2014.08.017
Ponomarova, O., and Patil, K. R. (2015). Metabolic interactions in microbial communities: untangling the Gordian knot. Curr. Opin. Microbiol. 27, 37-44. doi: 10.1016/j.mib.2015.06.014

Rein, A., Adam, I. K., Miltner, A., Brumme, K., Kästner, M., and Trapp, S. (2016). Impact of bacterial activity on turnover of insoluble hydrophobic substrates (phenanthrene and pyrene)-Model simulations for prediction of bioremediation success. J. Hazard. Mater. 306, 105-114. doi: 10.1016/j.jhazmat. 2015.12.005

Shoaie, S., Karlsson, F., Mardinoglu, A., Nookaew, I., Bordel, S., and Nielsen, J. (2013). Understanding the interactions between bacteria in the human gut through metabolic modeling. Sci. Rep. 3:2532. doi: 10.1038/srep02532

Song, H.-S., Cannon, W., Beliaev, A., and Konopka, A. (2014). Mathematical modeling of microbial community dynamics: a methodological review. Processes 2, 711-752. doi: 10.3390/pr2040711

Sonnenburg, J. L., Xu, J., Leip, D. D., Chen, C. H., Westover, B. P., Weatherford, J., et al. (2005). Glycan foraging in vivo by an intestine-adapted bacterial symbiont. Science 307, 1955-1959. doi: 10.1126/science.1109051

Steinway, S. N., Biggs, M. B., Loughran, T. P. Jr., Papin, J. A., and Albert, R. (2015). Inference of network dynamics and metabolic interactions in the gut microbiome. PLoS Comput. Biol. 11:e1004338. doi: 10.1371/journal.pcbi. 1004338

Stolyar, S., Van Dien, S., Hillesland, K. L., Pinel, N., Lie, T. J., Leigh, J. A., et al. (2007). Metabolic modeling of a mutualistic microbial community. Mol. Syst. Biol. 3:92. doi: 10.1038/msb4100131

Taffs, R., Aston, J. E., Brileya, K., Jay, Z., Klatt, C. G., Mcglynn, S., et al. (2009). In silico approaches to study mass and energy flows in microbial consortia: a syntrophic case study. BMC Syst. Biol. 3:114. doi: 10.1186/1752-0509-3-114

Tasoff, J., Mee, M. T., and Wang, H. H. (2015). An economic framework of microbial trade. PLoS ONE 10:e0132907. doi: 10.1371/journal.pone.0132907

Terahara, K., Nochi, T., Yoshida, M., Takahashi, Y., Goto, Y., Hatai, H., et al. (2011). Distinct fucosylation of M cells and epithelial cells by Fut1 and Fut2, respectively, in response to intestinal environmental stress. Biochem. Biophys. Res. Commun. 404, 822-828. doi: 10.1016/j.bbrc.2010.12.067

Theis, K. R., Dheilly, N. M., Klassen, J. L., Brucker, R. M., Baines, J. F., Bosch, T. C., et al. (2016). Getting the hologenome concept right: an eco-evolutionary framework for hosts and their microbiomes. mSystems 1:e00028-16. doi: 10.1128/mSystems.00028-16

Thiele, I., and Palsson, B. O. (2010). A protocol for generating a high-quality genome-scale metabolic reconstruction. Nat. Protoc. 5, 93-121. doi: 10.1038/ nprot.2009.203

Tobalina, L., Bargiela, R., Pey, J., Herbst, F. A., Lores, I., Rojo, D., et al. (2015). Context-specific metabolic network reconstruction of a naphthalene-degrading bacterial community guided by metaproteomic data. Bioinformatics 31, 1771-1779. doi: 10.1093/bioinformatics/btv036

Turnbaugh, P. J., Backhed, F., Fulton, L., and Gordon, J. I. (2008). Diet-induced obesity is linked to marked but reversible alterations in the mouse distal gut microbiome. Cell Host Microbe 3, 213-223. doi: 10.1016/j.chom.2008. 02.015

Tzamali, E., Poirazi, P., Tollis, I. G., and Reczko, M. (2011). A computational exploration of bacterial metabolic diversity identifying metabolic interactions and growth-efficient strain communities. BMC Syst. Biol. 5:167. doi: 10.1186/ 1752-0509-5-167

Varma, A., and Palsson, B. O. (1993). Metabolic capabilities of Escherichia coli: I. synthesis of biosynthetic precursors and cofactors. J. Theor. Biol. 165, 477-502. doi: 10.1006/jtbi.1993.1202

Werner, G. D., Strassmann, J. E., Ivens, A. B., Engelmoer, D. J., Verbruggen, E., Queller, D. C., et al. (2014). Evolution of microbial markets. Proc. Natl. Acad. Sci. U.S.A. 111, 1237-1244. doi: 10.1073/pnas.1315980111

West, S. A., Griffin, A. S., Gardner, A., and Diggle, S. P. (2006). Social evolution theory for microorganisms. Nat. Rev. Microbiol. 4, 597-607. doi: 10.1038/ nrmicro1461

Wood, D. E., and Salzberg, S. L. (2014). Kraken: ultrafast metagenomic sequence classification using exact alignments. Genome Biol. 15:R46. doi: 10.1186/gb2014-15-3-r46

Wyatt, G. A., Kiers, E. T., Gardner, A., and West, S. A. (2016). Restricting mutualistic partners to enforce trade reliance. Nat. Commun. 7:10322. doi: $10.1038 /$ ncomms10322 
Yatsunenko, T., Rey, F. E., Manary, M. J., Trehan, I., Dominguez-Bello, M. G., Contreras, M., et al. (2012). Human gut microbiome viewed across age and geography. Nature 486, 222-227. doi: 10.1038/nature11053

Ye, C., Zou, W., Xu, N., and Liu, L. (2014). Metabolic model reconstruction and analysis of an artificial microbial ecosystem for vitamin $\mathrm{C}$ production. J. Biotechnol. 182, 61-67. doi: 10.1016/j.jbiotec.2014.04.027

Zelezniak, A., Andrejev, S., Ponomarova, O., Mende, D. R., Bork, P., and Patil, K. R. (2015). Metabolic dependencies drive species co-occurrence in diverse microbial communities. Proc. Natl. Acad. Sci. U.S.A. 112, 6449-6454. doi: $10.1073 /$ pnas.1421834112

Zhuang, K., Izallalen, M., Mouser, P., Richter, H., Risso, C., Mahadevan, R., et al. (2011). Genome-scale dynamic modeling of the competition between Rhodoferax and Geobacter in anoxic subsurface environments. ISME J. 5, 305-316. doi: 10.1038/ismej.2010.117

Zomorrodi, A. R., and Maranas, C. D. (2012). OptCom: a multi-level optimization framework for the metabolic modeling and analysis of microbial communities. PLoS Comput. Biol. 8:e1002363. doi: 10.1371/journal.pcbi. 1002363

Zomorrodi, A. R., and Segre, D. (2016). Synthetic ecology of microbes: mathematical models and applications. J. Mol. Biol. 428, 837-861. doi: 10.1016/ j.jmb.2015.10.019

Conflict of Interest Statement: The authors declare that the research was conducted in the absence of any commercial or financial relationships that could be construed as a potential conflict of interest.

Copyright (C) 2017 Bosi, Bacci, Mengoni and Fondi. This is an open-access article distributed under the terms of the Creative Commons Attribution License (CC BY). The use, distribution or reproduction in other forums is permitted, provided the original author(s) or licensor are credited and that the original publication in this journal is cited, in accordance with accepted academic practice. No use, distribution or reproduction is permitted which does not comply with these terms. 